\title{
Special issue on PDE models and computation: part III
}

\author{
S. Sundar ${ }^{1}$
}

(C) Indian Institute of Technology Madras 2016

This issue is the third in the sequel of the Special Issue: "PDE Models and Computation". Five carefully selected high quality works are published here. The first four papers mainly focus on numerical treatments while the fifth paper is on a modelling problem.

I strongly believe that these well chosen papers put forth interesting and challenging research problems which, I am quite sure, would be useful to budding researchers.

Scheduled Relaxation Jacobi (SRJ) method, by Babu Viswanathan, is a promising numerical technique for the solution of the Poisson equation. This is due to the fact that the spectral radius of the method is improved by a proper choice of relaxation parameters and it is parallelizable.

A second order convergence vertex-based FVM for the Laplace operator on triangular grids with Dirichlet condition implemented weakly is a new line of thought by C.Praveen.

J.Mahipal et al. propose a new $5 \times 5$ stencil scheme based on generalized FDM. This is another interesting and useful idea which reduces the checkerboard artefacts in image denoising.

The flow induced by shrinking sheets and the associated heat and mass transfer are considered to be important in the understanding of extrusion processes. Sanantan Das et al. propose a numerical approach that transforms the governing nonlinear PDE model to a system of nonlinear ODE model, which in turn, is solved by a robust RK method.

Capturing mathematically, the scattering of membrane-coupled gravity waves by membrane surface and porous bottom variations in ocean waves is an important problem. The second order mild-slope model equation proposed by Ramana Babu and S.R.Manam is an intelligent idea that further investigates Bragg resonance on different variations.

A few more original contributions are in the pipeline. Keep yourself connected to AEAM and enjoy reading good research!

S. Sundar

slnt@iitm.ac.in

1 IIT, Madras, India 